\title{
Paraneoplastic Palmar Fasciitis and Polyarthritis Syndrome in a Metastatic Breast Cancer Patient: Rare Case Report
}

\begin{abstract}
Palmar fasciitis and polyarthritis syndrome (PFAPS) is a rare paraneoplastic event that has been reported mainly in ovarian cancers. Clinical diagnosis of PFAPS is characterized by the diagnosis of neoplasm with accompanying flexion contractures, inflammatory fasciitis, fibrosis, and generalized arthritis of the hands. Since first published report, only rare few additional cases of PFPAS have been reported. In this case report, we describe a patient with metastatic breast cancer who subsequently developed PFPAS in association with advanced breast cancer. She had clinical rheumatological symptoms course parallel to her primary breast cancer disease status.
\end{abstract}

Keywords: Metastatic breast cancer, palmar fasciitis and polyarthritis syndrome, paraneoplastic syndrome, steroids

\section{Introduction}

Palmar fasciitis and polyarthritis syndrome (PFAPS) is a rare paraneoplastic event that has been reported mainly in ovarian cancers. The term "PFAPS" was first described by Medsger et al. ${ }^{[1]}$ in 1982. PFPAS is a challenging clinical diagnosis characterized by the diagnosis of neoplasm with accompanying flexion contractures, inflammatory fasciitis, fibrosis, and generalized arthritis of the hands. Since that seminal report published, only rare few additional cases of PFPAS have been reported, and these have been associated with a number of different neoplasms ranging from gynecologic malignancies (ovary, fallopian tubes), carcinoma pancreas, lung, breast carcinoma, prostate carcinoma to hematological malignancies, etc. ${ }^{[2]}$

In this case report, we describe a patient with metastatic breast cancer who developed PFPAS during her disease progression. She had clinical rheumatological symptoms course parallel to her primary breast cancer status.

\section{Case Report}

A 52-year-old North Indian woman was evaluated at our hospital in February 2015

\footnotetext{
This is an open access journal, and articles are distributed under the terms of the Creative Commons AttributionNonCommercial-ShareAlike 4.0 License, which allows others to remix, tweak, and build upon the work non-commercially, as long as appropriate credit is given and the new creations are licensed under the identical terms.
}

For reprints contact: reprints@medknow.com for lump in bilateral breasts. Local examination revealed breast lump of $3 \mathrm{~cm} \times 3 \mathrm{~cm}$ in upper outer quadrant of the right side and $3 \mathrm{~cm} \times 4 \mathrm{~cm}$ lesion in upper inner quadrant of the left breast. Mammography revealed BIRADS IV bilaterally. Tru-cut biopsy from both breasts revealed infiltrating ductal carcinoma. Immunohistochemistry (IHC) from right breast biopsy revealed estrogen receptor $8 / 8$, progesterone receptor $8 / 8$, Her $2 /$ neu - negative, and $\mathrm{Ki}-67 \%-35 \%$. IHC from left breast showed triple negative breast cancer, $\mathrm{Ki}-67 \%-15 \%$. On positron emission tomography-computed tomography (PET-CT) fluorodeoxyglucose avid bony metastasis along with bilateral breast lesions were present. The patient did not have any rheumatological diagnosis or complaints at the time of presentation.

The patient was started on palliative chemotherapy (docetaxel + capecitabine) from February 2015. Post 4 cycles chemotherapy, PET-CT revealed partial response and then, she was started on hormonal therapy (tamoxifen + pamorelin). The patient had clinical progression on hormonal therapy in September 2015, hence was started on the second line chemotherapy (NAB paclitaxel + carboplatin). She showed good response to therapy as clinically no mass was palpable in the right breast and left lesion was shrunk. How to cite this article: Sehrawat A, Rao T,
Sharma L, Parthasarathy KM. Paraneoplastic palmar
fasciitis and polyarthritis syndrome in a metastatic
breast cancer patient: Rare case report. Indian J Med
Paediatr Oncol 2019;40:570-2.

\author{
Amit Sehrawat ${ }^{1}$, \\ Trivikram Rao', \\ Lalit Sharma², \\ KM Parthasarathy ${ }^{2}$ \\ ${ }^{1}$ Department of Medical \\ Oncology and Haematology, \\ All India Institute of \\ Medical Sciences, Rishikesh, \\ Uttarakhand, ${ }^{2}$ Department of \\ Medical Oncology, Dharamshila \\ Narayana Superspeciality \\ Hospital, New Delhi, India
}

Submission: 01-Jan-2018 Accepted in Revised Form: 19-Apr-2018

Published: 17-Feb-2020

Address for correspondence:

Dr. Amit Sehrawat, Dharamshila Narayana Superspeciality Hospital, Vasundhara Enclave, New Delhi - 110 096, India. E-mail: dramitsehrawat@ gmail.com

Access this article online

Website: www.ijmpo.org

DOI: 10.4103/ijmpo.ijmpo_1_18 Quick Response Code:

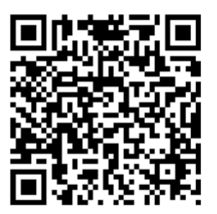


However, patient defaulted follow-up and presented 5 months later with progression in the form of left supraclavicular lymphnode in March 2016. At the same time, she began to experience symptoms of polyarthritis with bilateral pain and swelling and stiffness in her hands, wrists, knees, ankles, and feet which were more in the early morning, not regressing after rest [Figure 1]. She also noticed thickening and erythema of her palmar skin followed by rapid progression to flexion contractures in her both hands. She also noticed tingling and numbness in all four limbs. Physical examination revealed synovitis of the metacarpophalangeal (MCP) and proximal interphalangeal (PIP) joints with marked restriction of movement, and the skin of the palms was leathery and rock-hard to palpation consistent with palmar fasciitis [Figure 2]. The patient had pain and decreased the range of motion in her elbows, knees, ankles, and feet. The patient showed no signs of Raynaud's phenomenon. The rheumatologic consultation was done, the results of laboratory tests, including full blood count, antinuclear antibody (ANA), uric acid and an antibody screen (anti-double-stranded DNA, anticentromeric protein, anti-extractable nuclear antigen, anti-topoisomerase I, anti-cyclic citrullinated protein) were all unremarkable. While erythrocyte sedimentation rate (ESR) was high at $35 \mathrm{~mm} / \mathrm{h}$. Plain-film X-rays of the hands showed no findings of acute bony, soft-tissue, or joint space abnormalities. A bone scan was consistent with an inflammatory polyarthropathy, without evidence of osseous metastasis. Nerve conduction studies done were normal. A clinical diagnosis of PFPAS was made. Although this syndrome is often resistant to anti-inflammatory and anti-rheumatic therapies, oral steroids were initiated. She was continued on NAB-paclitaxel based palliative chemotherapy, her rheumatological and cancer-related symptoms improved for a short duration of about 2 months.

Subsequently, her PET-CT showed progressive disease which was accompanied by joint symptoms progression. She was then started on the $3^{\text {rd }}$ line chemotherapy (Eribulin based), but still her disease progressed as documented by PET-CT post 4 cycles chemotherapy

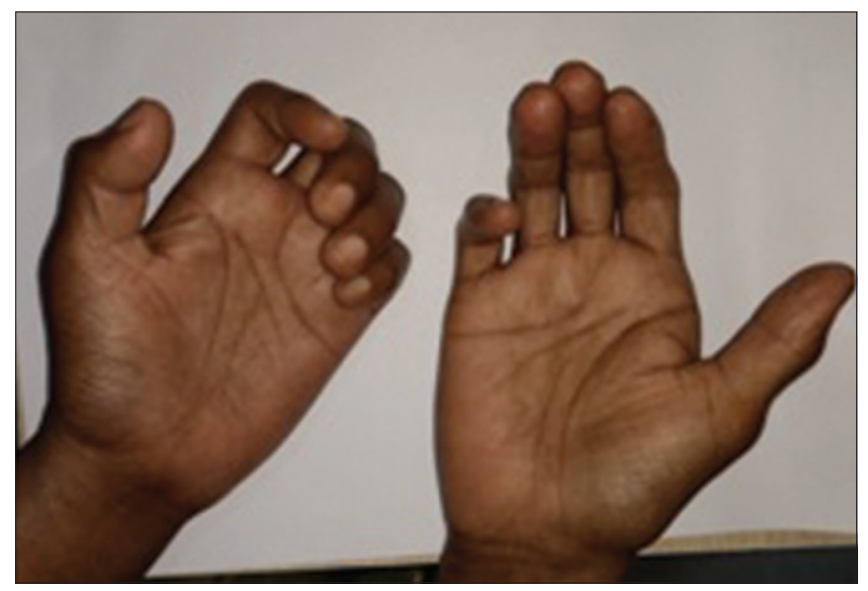

Figure 1: Paraneoplastic palmar fasciitis and polyarthritis syndrome leading to contractures and flexion deformities and with that she also had rheumatological symptom progression.

After multiple lines of palliative treatment, she continued to deteriorate both in terms of breast cancer and PFPAS and finally expired on March 2017.

\section{Discussion}

Paraneoplastic syndromes are disorders caused by systemic effects occurring remotely from the cancer primary site or metastasis. They are estimated to affect up to $8 \%$ of patients with cancer. ${ }^{[3]}$ Paraneoplastic syndromes are cancer-associated diseases or symptoms that occur distant from the underlying malignancy. They are not directly caused by a tumor or its metastases but are mediated by humoral factors, such as hormones and cytokines, or they are a consequence of the immune response against tumor cells.

Musculoskeletal problems associated with malignancy include (i) arthropathies, (ii) muscular disorders including dermatomyositis and polymyositis, (iii) scleroderma, panniculitis and fasciitis, (iv) vasculitides, and (v) miscellaneous rheumatic syndromes. ${ }^{[4]}$ PFPAS is a rare paraneoplastic musculoskeletal condition associated with malignancy and is usually seen in association with ovarian cancer or other gynecologic malignancies. ${ }^{[1]}$

In most of the reported cases, PFPAS presents with a sudden onset of diffuse painful swelling of both the hands with marked stiffness. Later nodular thickening of the palmar fascia develops similar to Dupuytren's contracture, but much more severe. In $36.8 \%$ of women, by far the most frequent tumor type in paraneoplastic PFPAS is ovarian adenocarcinoma. ${ }^{[5,6]}$ Ovarian and breast cancer together with malignancies of other female reproductive organs are the underlying cause in more than half of all published cases. About 10 patients with PFPAS in carcinoma breast have been reported so far as per PubMed database. We here report one of such patients. Overall, $20 \%$ of the reported cases exhibit a similar involvement of the plantar

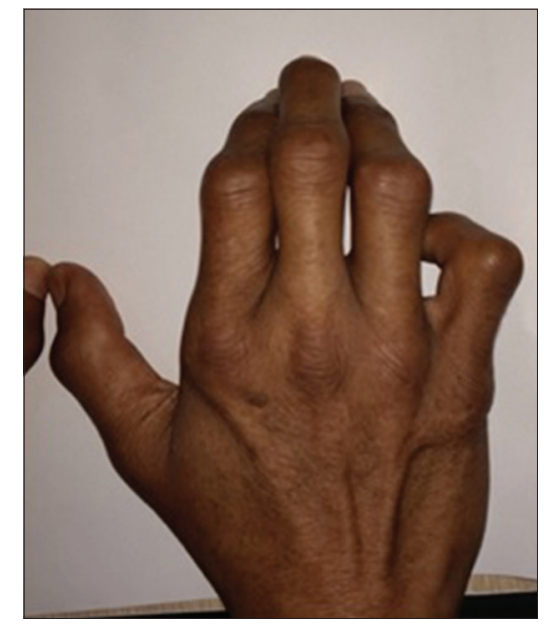

Figure 2: Palmar fasciitis leading to severe flexion deformities 
fascia. Some authors describe the palpatory findings in an advanced stage with the illustrative term "woody hands."[7]

The underlying mechanism for paraneoplastic PFPAS is currently unknown. The majority of all paraneoplastic PFPAS patients experience the onset of symptoms between the sixth and eighth decade of life. PFPAS has been described in females more than four times more frequent than in males. This observation has often been used as an argument for a pathophysiological role of female sex hormones in the pathogenesis of this paraneoplastic syndrome but that has not been proven yet. ${ }^{[7]}$ However, it is likely that fibrogenic and connective tissue growth factors and autoimmune mechanisms produced by the neoplasm are responsible for the development of PFPAS because complement and immunoglobulin deposits have been found in some of the fascial tissues. There has been no direct or consistent link with any particular autoantibodies. However, the ESR is usually elevated, ANA is often weakly positive, and rheumatoid factor (RF) is almost always negative. ${ }^{[2]}$

Conventionally, the severity of paraneoplastic syndromes is unrelated to the size of the primary tumor or the extent of metastatic disease. In most cases of PFPAS, the rheumatologic symptoms preceded the detection of the underlying malignancy, but this was not the case here. Exacerbation of inflammatory joint symptoms during subsequent relapse/progression of the underlying malignancy may occur as in the present case.

Clinical features of PFPAS include pain and diffuse synovitis of the hands (usually at the MCP and PIP joints) and a symmetric polyarthritis with rapid progression of palmar fasciitis with flexion contractures of the hands. Unlike the scleroderma-related syndromes (which this condition may mimic), patients with PFPAS universally lack evidence of sclerodactyly. The development of symptoms (especially in the palms and hands) is usually rapid, leaving the patient severely disabled in several days or weeks.

Laboratory tests, such as acute-phase reactants and the presence of antibodies, generally provide unremarkable results. ANA and rarely RF are the most common antibodies detected in PFPAS cases, suggesting a potential role for autoimmunity; PFPAS seems to be a seronegative disorder. However, to further differentiate true paraneoplastic rheumatic syndromes from the coincidental co-occurrence of rheumatologic symptoms with cancer, autoantibodies may contribute to differentiate the two entities.

The histopathologic abnormalities in PFPAS are nonspecific and have unclear diagnostic value. Biopsies may reveal features of nodular fasciitis, mononuclear, or lymphocytic infiltrates, but biopsy is not generally recommended to confirm the diagnosis, which is usually made on clinical grounds. ${ }^{[2]}$

Corticosteroids are often used as a first-line therapy. The efficacy of systemic steroids in patients with PFPAS is somewhat disappointing, similar results have been largely disappointing with the use of conventional disease-modifying anti-rheumatic drugs, more recently, tumor necrosis factor (TNF) inhibitors have been attempted as a second-line treatment or in combination with corticosteroids. However, of particular concern for patients with cancer are the immunosuppressive effects of anti-TNF antibody therapies and their potential effect on accelerating cancer progression. ${ }^{[2]}$

\section{Conclusion}

PFPAS is a rare paraneoplastic syndrome that usually occurs in women, and that is characterized by a nonerosive polyarthritis and fibrosis of the palmar fascia, resulting in contractures of the hands. We presented a case of PFPAS associated with advanced breast cancer and described an apparent acceleration of cancer progression during therapy. The fact that PFPAS is generally resistant to anti-inflammatory and anti-rheumatic therapies emphasizes the importance of early diagnosis and treatment of the underlying malignancy to prevent the development of permanent flexion contractures.

\section{Declaration of patient consent}

The authors certify that they have obtained all appropriate patient consent forms. In the form the patient(s) has/have given his/her/their consent for his/her/their images and other clinical information to be reported in the journal. The patients understand that their names and initials will not be published and due efforts will be made to conceal their identity, but anonymity cannot be guaranteed.

\section{Financial support and sponsorship}

Nil.

\section{Conflicts of interest}

There are no conflicts of interest.

\section{References}

1. Medsger TA, Dixon JA, Garwood VF. Palmar fasciitis and polyarthritis associated with ovarian carcinoma. Ann Intern Med 1982;96:424-31.

2. Nadal R, McMahan ZH, Antonarakis ES. Paraneoplastic palmar fasciitis and polyarthritis syndrome in a patient with advanced prostate cancer. Clin Genitourin Cancer 2013;11:e15-23.

3. Pelosof LC, Gerber DE. Paraneoplastic syndromes: An approach to diagnosis and treatment. Mayo Clin Proc 2010;85:838-54.

4. Naschitz JE, Rosner I. Musculoskeletal syndromes associated with malignancy (excluding hypertrophic osteoarthropathy). Curr Opin Rheumatol 2008;20:100-5.

5. Santra G. Paraneoplastic palmar fasciitis and polyarthritis syndrome. J Assoc Physicians India 2009;57:79-81.

6. Qureshi AA, Saavedra A. Palmar fasciitis and polyarthritis syndrome in patients with ovarian cancer - A case report and review of the literature. Hand (N Y) 2011;6:220-3.

7. Manger B, Schett G. Palmar fasciitis and polyarthritis syndrome-systematic literature review of 100 cases. Semin Arthritis Rheum 2014;44:105-11. 